\title{
Gevrey class regularity for analytic differential-delay equations
}

\author{
Roger D. Nussbaum ${ }^{1}$ and Gabriella Vas ${ }^{\bowtie 2}$ \\ ${ }^{1}$ Department of Mathematics, Hill Center, Rutgers University, \\ 110 Frelinghuysen Rd., Piscataway, NJ 08854-8019, USA \\ ${ }^{2}$ MTA-SZTE Analysis and Stochastics Research Group, Bolyai Institute, University of Szeged, \\ 1 Aradi vértanúk tere, Szeged, H-6720, Hungary
}

Appeared 11 August 2016

Communicated by Tibor Krisztin

\begin{abstract}
This paper considers differential-delay equations of the form

$$
x^{\prime}(t)=p(t) x(t-1),
$$

where the coefficient function $p: \mathbb{R} \rightarrow \mathbb{C}$ is analytic and not bounded on any $\delta$-neighborhood of the intervals $(-\infty, \gamma], \gamma \in \mathbb{R}$. For these equations, we cannot apply the known results regarding the analyticity of the bounded solutions $x:(-\infty, \gamma] \rightarrow \mathbb{C}$. We prove Gevrey class regularity for such solutions.
\end{abstract}

Keywords: delay equation, analyticity, Gevrey class.

2010 Mathematics Subject Classification: 34K06, 34K99.

\section{Introduction}

The analyticity of globally defined bounded solutions of autonomous analytic delay equations was studied first in [6]. The result of [6] was generalized to the nonautonomous case in [4]. Paper [4] verifies that if $\gamma \in \mathbb{R}, x:(-\infty, \gamma] \rightarrow \mathbb{C}^{n}$ is a bounded, uniformly continuous solution of

$$
x^{\prime}(t)=f\left(t, x_{t}\right)
$$

on the interval $(-\infty, \gamma]$, and $f$ is analytic and bounded on a $\delta$-neighborhood of the set $\left\{\left(t, x_{t}\right): t \in(-\infty, \gamma]\right\}$, then $x$ is real analytic, i.e., there exists an open neighborhood $V$ of $(-\infty, \gamma]$ and a complex analytic map $\hat{x}: V \rightarrow \mathbb{C}^{n}$ such that $\left.\hat{x}\right|_{(-\infty, \gamma]}=x$. It is an interesting question whether the condition regarding the boundedness of $f$ can be relaxed.

The result of [4] is not applicable to equations of the form

$$
x^{\prime}(t)=p(t) x(t-1)
$$

\footnotetext{
${ }^{\bowtie}$ Corresponding author. Email: vasg@math.u-szeged.hu
} 
if $p$ is analytic but not bounded on any $\delta$-neighborhood of $(-\infty, \gamma]$. Typical examples of such coefficient functions are $p(t)=e^{i t^{q}}$ and $p(t)=\sin \left(t^{q}\right)$ with an integer $q \geq 2$. In this paper we investigate the case when

$$
p(t)=\sum_{m \in F} A_{m} e^{i m \omega t^{q}}, \quad t \in \mathbb{R}
$$

$F$ is a finite set of integers, $A_{m} \in \mathbb{C}$ for $m \in F, \omega>0, i=\sqrt{-1}, A_{0}=0$ and $q \geq 2$ is an integer. We know from [5] that for such coefficient functions and for any $c \in \mathbb{C} \backslash\{0\}$, there exists a $C^{\infty}$ function $x$ such that $x$ satisfies the equation (1.1) for all $t \in \mathbb{R}$ and $\lim _{t \rightarrow-\infty} x(t)=c$. Is this solution analytic at any $t_{0} \in \mathbb{R}$ ? We conjecture that the answer is negative. We can prove that $x$ is of Gevrey class.

Gevrey classes are intermediate spaces between the spaces of $C^{\infty}$ functions and real analytic functions. Let $J$ be a nonempty, open subset of $\mathbb{R}$. Let $q>1$. We say that $x: J \rightarrow \mathbb{C}$ is of Gevrey class $q$ in $J$ if for each compact set $K \subset J$, there exists a constant $C_{K}$ such that

$$
\left|x^{(n)}(t)\right| \leq C_{K}^{n+1}(n !)^{q}
$$

for all $t \in K$ and for all nonnegative integer $n$ [1]. In this work $J=\mathbb{R}$.

Gevrey classes play a prominent role in the theory of partial differential equations; but, to the best of our knowledge, they have not previously been studied in connection with differential-delay equations. Our results below suggest that further work in this direction may be appropriate.

Theorem 1.1. Let

$$
p(t)=\sum_{m \in F} A_{m} e^{i m \omega t^{q}}, \quad t \in \mathbb{R}
$$

where $F$ is a finite set of integers, $A_{m} \in \mathbb{C}$ for $m \in F, \omega>0, i=\sqrt{-1}, A_{0}=0$ and $q \geq 2$ is an integer. Suppose that $x: \mathbb{R} \rightarrow \mathbb{C}$ satisfies (1.1) for each $t \in \mathbb{R}$, and $x$ has a nonzero limit as $t \rightarrow-\infty$. Then $x$ is of Gevrey class $q$ in $\mathbb{R}$.

The question of analyticity is even more interesting for delay equations with timedependent or state-dependent delays. Mallet-Paret and Nussbaum have constructed a timedependent delay equation in [3] such that a given solution is analytic at certain points of its domain and nonanalytic at others. Krisztin has shown analyticity for a particular class of equations with state-dependent delay in [2]. As far as we know, this is the only positive result in the state-dependent delay case.

We close the paper by showing that in general we cannot expect the nonanalytic solutions of analytic equations to admit Gevrey regularity. We consider the linear inhomogeneous equation

$$
x^{\prime}(t)=a(t) x(t)+b(t) x(\eta(t))+h(t)
$$

from [3], where $a, b, h$ and $\eta$ are analytic in $t$ in a neighborhood of $t=t_{0} \in \mathbb{R}$. We assume that $t_{0}$ is an expansive fixed point of $\eta$, i.e., $\eta\left(t_{0}\right)=t_{0}$ and $\left|\eta^{\prime}\left(t_{0}\right)\right|>1$. Let $x_{0} \in \mathbb{R}$ be given. The paper [3] gives a mild technical condition under which equation (1.2) with initial value $x\left(t_{0}\right)=x_{0}$ has no analytic solution in any open neighborhood of $t=t_{0}$. Using the results of [3], we easily show at the end of this paper that such solutions are not of Gevrey class $q$ for any $q>1$ either. 


\section{The proof of Theorem 1.1}

The proof of the theorem relies on two lemmas and estimates on the derivatives of the coefficient function $p$.

Recall that by the product rule,

$$
\left(f_{1}(t) f_{2}(t)\right)^{(n)}=\sum_{i=0}^{n}\left(\begin{array}{c}
n \\
i
\end{array}\right) f_{1}^{(n-i)}(t) f_{2}^{(i)}(t)
$$

for all $n \in \mathbb{N}=\{0,1,2, \ldots\}, f_{1} \in C^{n}(\mathbb{R}, \mathbb{C}), f_{2} \in C^{n}(\mathbb{R}, \mathbb{C})$ and $t \in \mathbb{R}$. Hence for any solution $x: \mathbb{R} \rightarrow \mathbb{C}$ of equation (1.1), $t \in \mathbb{R}$ and $n \in \mathbb{N}$ with $n \geq 1$,

$$
x^{(n)}(t)=\sum_{i=0}^{n-1}\left(\begin{array}{c}
n-1 \\
i
\end{array}\right) p^{(n-1-i)}(t) x^{(i)}(t-1) .
$$

We use this observation to express $x^{(n)}(t), n \geq 1, t \in \mathbb{R}$, as a function of the values $x(t-k)$, $k \in\{1, \ldots, n\}$, and the derivatives of $p$ at $t-l$, where $l \in\{0, \ldots, n-1\}$.

For all $n \geq 1$ and $1 \leq k \leq n$, let $\sum_{(n, k)}$ denote the sum taken over the elements of the set

$$
S_{n, k}=\left\{\left(j_{0}, j_{1}, \ldots, j_{k}\right) \in \mathbb{N}^{k+1}: n=j_{0}>j_{1}>\cdots>j_{k}=0\right\} .
$$

Lemma 2.1. Assume that $x: \mathbb{R} \rightarrow \mathbb{C}$ satisfies equation (1.1) on $\mathbb{R}$. Then for all $t \in \mathbb{R}$ and $n \geq 1$,

$$
x^{(n)}(t)=\sum_{k=1}^{n} q_{n, k}(t) x(t-k),
$$

where

$$
q_{n, k}(t)=n ! \sum_{(n, k)} \prod_{l=0}^{k-1} \frac{p^{\left(j_{l}-1-j_{l+1}\right)}(t-l)}{j_{l}\left(j_{l}-1-j_{l+1}\right) !}
$$

for all $t \in \mathbb{R}, n \geq 1$ and $1 \leq k \leq n$.

Note that by Lemma 2.1,

$$
q_{n, 1}(t)=p^{(n-1)}(t) \quad \text { and } \quad q_{n, n}(t)=\prod_{l=0}^{n-1} p(t-l) \quad \text { for } t \in \mathbb{R} \text { and } n \geq 1 .
$$

Proof. It is clear that $x^{(n)}(t)$ exists for all $t \in \mathbb{R}$ and $n \geq 1$.

The proof goes by induction on $n$. By definition, $q_{11}(t)=p(t)$ for all real $t$, hence the assertion holds for all $t \in \mathbb{R}$ and $n=1$. Let $n \geq 2$ and suppose the lemma holds for all $t \in \mathbb{R}$ and $i \in \mathbb{N}$ with $1 \leq i<n$. Then applying (2.1) and our induction hypothesis, we deduce that

$$
\begin{aligned}
x^{(n)}(t) & =\sum_{i=0}^{n-1}\left(\begin{array}{c}
n-1 \\
i
\end{array}\right) p^{(n-1-i)}(t) x^{(i)}(t-1) \\
& =p^{(n-1)}(t) x(t-1)+\sum_{i=1}^{n-1}\left(\begin{array}{c}
n-1 \\
i
\end{array}\right) p^{(n-1-i)}(t) \sum_{k=1}^{i} q_{i, k}(t-1) x(t-1-k) \\
& =p^{(n-1)}(t) x(t-1)+\sum_{i=1}^{n-1}\left(\begin{array}{c}
n-1 \\
i
\end{array}\right) p^{(n-1-i)}(t) \sum_{k^{\prime}=2}^{i+1} q_{i, k^{\prime}-1}(t-1) x\left(t-k^{\prime}\right) \\
& =p^{(n-1)}(t) x(t-1)+\sum_{k=2}^{n} \sum_{i=k-1}^{n-1}\left(\begin{array}{c}
n-1 \\
i
\end{array}\right) p^{(n-1-i)}(t) q_{i, k-1}(t-1) x(t-k) .
\end{aligned}
$$


Let

$$
\hat{q}_{n, k}(t)=\sum_{i=k-1}^{n-1}\left(\begin{array}{c}
n-1 \\
i
\end{array}\right) p^{(n-1-i)}(t) q_{i, k-1}(t-1), \quad t \in \mathbb{R}, k \in\{2, \ldots, n\} .
$$

As $p^{(n-1)} \equiv q_{n, 1}$ by definition, we need to show that $\hat{q}_{n, k} \equiv q_{n, k}$ for all $k \in\{2, \ldots, n\}$.

Formula (2.3) and the substitutions $j_{l}^{\prime}=j_{l-1}, l \in\{1, \ldots, k\}$, give that

$$
q_{i, k-1}(t-1)=i ! \sum_{\left(j_{1}^{\prime}, \ldots, j_{k}^{\prime}\right) \in S_{i, k-1}} \prod_{l=1}^{k-1} \frac{p^{\left(j_{l}^{\prime}-1-j_{l+1}^{\prime}\right)}(t-l)}{j_{l}^{\prime}\left(j_{l}^{\prime}-1-j_{l+1}^{\prime}\right) !} .
$$

Recall that $j_{1}^{\prime}=i$ in the above expression. Substituting $i$ for $j_{1}^{\prime}$ in (2.4), we see that

$$
\hat{q}_{n, k}(t)=n ! \sum_{j_{1}^{\prime}=k-1}^{n-1} \frac{p^{\left(n-1-j_{1}^{\prime}\right)}(t)}{n\left(n-1-j_{1}^{\prime}\right) !} \sum_{\left(j_{1}^{\prime}, \ldots, j_{k}^{\prime}\right) \in S_{i, k-1}} \prod_{l=1}^{k-1} \frac{p^{\left(j_{l}^{\prime}-1-j_{l+1}^{\prime}\right)}(t-l)}{j_{l}^{\prime}\left(j_{l}^{\prime}-1-j_{l+1}^{\prime}\right) !} .
$$

It is clear that $\left(n, j_{1}^{\prime}, \ldots, j_{k}^{\prime}\right) \in S_{n, k}$ if and only if

$$
k-1 \leq j_{1}^{\prime} \leq n-1 \quad \text { and } \quad\left(j_{1}^{\prime}, \ldots, j_{k}^{\prime}\right) \in S_{i, k-1} .
$$

Writing $j_{l}$ instead of $j_{l}^{\prime}$, this means that

$$
\hat{q}_{n, k}(t)=n ! \sum_{(n, k)} \prod_{l=0}^{k-1} \frac{p^{\left(j_{l}-1-j_{l+1}\right)}(t-l)}{j_{l}\left(j_{l}-1-j_{l+1}\right) !}=q_{n, k}(t)
$$

for all $k \in\{2, \ldots, n\}$ and $t \in \mathbb{R}$, and the proof is complete.

We obtain the following as a consequence.

Lemma 2.2. Suppose there exist $q>1, C \geq 1$ and $t_{0} \in \mathbb{R}$ such that

$$
\left|p^{(n)}(t)\right| \leq C^{n+1}(\max (|t|, 1))^{(q-1) n} n ! \quad \text { for } t \leq t_{0} \text { and } n \in \mathbb{N} .
$$

Let $x: \mathbb{R} \rightarrow \mathbb{C}$ be a solution of equation (1.1) on $\mathbb{R}$ such that $|x(t)| \leq M$ for all $t \leq t_{0}$. Then

$$
\left|x^{(n)}(t)\right| \leq M(2 C)^{n}(|t|+n)^{(q-1) n} n ! \quad \text { for all } t \leq t_{0} \text { and } n \in \mathbb{N} .
$$

Proof. By assumption we have $|x(t)| \leq M(2 C)^{0}(\max (|t|, 1))^{(q-1) 0} 0$ ! for all $t \leq t_{0}$.

Fix $n \geq 1$ and $t \leq t_{0}$. According to Lemma 2.1,

$$
x^{(n)}(t)=\sum_{k=1}^{n} q_{n, k}(t) x(t-k)
$$

where the coefficient functions $q_{n, k}, k \in\{1, \ldots, n\}$, are defined by (2.2) and (2.3). The estimate (2.6) implies that

$$
\left|q_{n, k}(t)\right| \leq n ! \sum_{(n, k)} \prod_{l=0}^{k-1} \frac{C^{j_{l}-j_{l+1}}(\max (|t-l|, 1))^{(q-1)\left(j_{l}-1-j_{l+1}\right)}}{j_{l}}
$$

for all $1 \leq k \leq n$. Notice that

$$
\max (|t-l|, 1) \leq|t|+k \text { for any } k \geq 1 \text { and } 0 \leq l \leq k-1 .
$$


Observe that

$$
\left|S_{n, k}\right|=\left(\begin{array}{l}
n-1 \\
k-1
\end{array}\right) \quad \text { for all } 1 \leq k \leq n
$$

moreover,

$$
\sum_{l=0}^{k-1} j_{l}-1-j_{l+1}=j_{0}-j_{k}-k=n-k \text { and } \prod_{l=0}^{k-1} j_{l} \geq n(k-1) !
$$

hold for all $1 \leq k \leq n$ and $\left(j_{0}, j_{1}, \ldots, j_{k}\right) \in S_{n, k}$.

Hence

$$
\begin{aligned}
\left|q_{n, k}(t)\right| & \leq(n-1) ! \sum_{(n, k)} \frac{1}{(k-1) !} C^{n}(|t|+k)^{(q-1)(n-k)} \\
& =(n-1) !\left|S_{n, k}\right| \frac{1}{(k-1) !} C^{n}(|t|+k)^{(q-1)(n-k)} \\
& =(n-1) !\left(\begin{array}{c}
n-1 \\
k-1
\end{array}\right) \frac{1}{(k-1) !} C^{n}(|t|+k)^{(q-1)(n-k)}
\end{aligned}
$$

for all $1 \leq k \leq n$, and

$$
\left|x^{(n)}(t)\right| \leq \sum_{k=1}^{n}\left|q_{n, k}(t)\right||x(t-k)| \leq M C^{n}(n-1) ! \sum_{k=1}^{n}\left(\begin{array}{l}
n-1 \\
k-1
\end{array}\right) \frac{(|t|+k)^{(q-1)(n-k)}}{(k-1) !} .
$$

If we note that

$$
\left(\begin{array}{l}
n-1 \\
k-1
\end{array}\right) \frac{1}{(k-1) !} \leq\left(\begin{array}{l}
n-1 \\
k-1
\end{array}\right) \leq 2^{n-1}
$$

and $(|t|+k)^{(q-1)(n-k)} \leq(|t|+n)^{(q-1) n}$ for $1 \leq k \leq n$, we obtain from (2.7) that

$$
\left|x^{(n)}(t)\right| \leq M C^{n}(n-1) ! n\left(2^{n-1}\right)(|t|+n)^{(q-1) n} \leq M(2 C)^{n} n !(|t|+n)^{(q-1) n} .
$$

Remark 2.3. One might hope that by a more careful exploitation of inequality (2.7), one could improve the estimate in Lemma 2.2 for $x^{(n)}(t)$, but this does not seem to be true. Let $\varepsilon \in(0,1 / 2)$. If $n$ is large enough, one can give a lower estimate for the sum

$$
M C^{n}(n-1) ! \sum_{k=1}^{n}\left(\begin{array}{l}
n-1 \\
k-1
\end{array}\right) \frac{(|t|+k)^{(q-1)(n-k)}}{(k-1) !}
$$

by considering only the $k^{\text {th }}$ term

$$
M C^{n}(n-1) !\left(\begin{array}{l}
n-1 \\
k-1
\end{array}\right) \frac{(|t|+k)^{(q-1)(n-k)}}{(k-1) !}=M C^{n} n ! \frac{k^{2}}{n^{2}} \frac{n !}{(k !)^{2}(n-k) !}(|t|+k)^{(q-1)(n-k)},
$$

where $1 \leq(\varepsilon / 2) n \leq k \leq \varepsilon n$. Recall that if $m$ is a positive integer, Stirling's formula asserts that there is a real number $\lambda(m) \in(0,1)$ such that

$$
m !=\sqrt{2 \pi m}\left(\frac{m}{e}\right)^{m} e^{\frac{\lambda(m)}{12 m}} .
$$

Using this, we see that

$$
\frac{n !}{(k !)^{2}(n-k) !}=\frac{\sqrt{n}}{2 \pi k \sqrt{n-k}} \frac{n^{n}}{k^{2 k}(n-k)^{n-k}} \exp \left(k+\frac{\lambda(n)}{12 n}-\frac{\lambda(k)}{6 k}-\frac{\lambda(n-k)}{12(n-k)}\right) .
$$


As $(\varepsilon / 2) n \leq k \leq \varepsilon n$ and $\lambda(m) \in(0,1)$ for all $m \geq 1$, there exist a constant $C^{*}>0$ independent of $n$ and $k$ such that

$$
\begin{aligned}
\frac{n !}{(k !)^{2}(n-k) !} & \geq C^{*} \frac{\sqrt{n}}{\varepsilon n \sqrt{n(1-\varepsilon / 2)}} \frac{n^{n}}{(\varepsilon n)^{2 \varepsilon n}(n(1-\varepsilon / 2))^{n(1-\varepsilon / 2)}} \\
& =C^{*} \frac{1}{n^{1+3 \varepsilon n / 2}} \frac{1}{\varepsilon^{1+2 \varepsilon n}}\left(1-\frac{\varepsilon}{2}\right)^{-1 / 2-n(1-\varepsilon / 2)}
\end{aligned}
$$

In addition,

$$
\frac{k^{2}}{n^{2}} \geq\left(\frac{\varepsilon}{2}\right)^{2} \text { and } \quad(|t|+k)^{(q-1)(n-k)} \geq\left(\frac{\varepsilon}{2}\right)^{(q-1)(1-\varepsilon / 2) n} n^{(q-1)(1-\varepsilon) n} .
$$

We conclude that there are constants $C_{1}>0$ and $C_{2}>0$ independent of $n$ such that the expression (2.9) (and thus (2.8)) is not smaller than

$$
M C^{n} n ! C_{1}\left(C_{2}\right)^{n} n^{(q-1)(1-\varepsilon) n-1-3 \varepsilon n / 2}
$$

for each $n \in \mathbb{N}$ and $t \leq t_{0}$.

Consider the case when $p(t)=e^{i t^{q}}$ for all real $t$ with an integer $q \geq 2$. Our next objective is to give a formula for $p^{(n)}(t)$ for each $n \in \mathbb{N}$ and $t \in \mathbb{R}$.

For each $u \in \mathbb{C}$,

$$
u^{q}-t^{q}=\prod_{k=0}^{q-1}\left(u-\eta_{k} t\right), \quad \text { where } \eta_{k}=e^{\frac{2 \pi i k}{q}}, k \in\{0,1, \ldots, q-1\} \text { and } i=\sqrt{-1} .
$$

It follows that

$$
e^{i\left(u^{q}-t^{q}\right)}=\sum_{j=0}^{\infty} \frac{\left(i\left(u^{q}-t^{q}\right)\right)^{j}}{j !}=\sum_{j=0}^{\infty} \frac{i^{j}}{j !} \prod_{k=0}^{q-1}\left(u-\eta_{k} t\right)^{j} .
$$

For each $j \geq 0$, define a set $R_{n, q, j}$ of $q$-tuples as

$$
R_{n, q, j}=\left\{\left(l_{0}, l_{1}, \ldots, l_{q-1}\right) \in \mathbb{N}^{q}: \sum_{k=0}^{q-1} l_{k}=n, l_{0}=j, 0 \leq l_{k} \leq j \text { for } 1 \leq k \leq q-1\right\} .
$$

Let $\sum^{(n, q, j)}$ denote the sum taken over the elements of $R_{n, q, j}$. Let $D_{t}^{n}$ denote the $n$-fold differentiation with respect to $t$.

Note that $\eta_{0}=1$ and $\eta_{k} \neq 1$ if $1 \leq k \leq q-1$. This observation and the product rule for higher order derivatives together give that

$$
\begin{aligned}
\left.D_{u}^{n} \prod_{k=0}^{q-1}\left(u-\eta_{k} t\right)^{j}\right|_{u=t} & =\sum^{(n, q, j)} \frac{n !}{l_{0} ! l_{1} ! \ldots l_{q-1} !} \prod_{k=0}^{q-1} \frac{j !}{\left(j-l_{k}\right) !}\left(t-\eta_{k} t\right)^{j-l_{k}} \\
& =n ! t^{q j-n} \sum^{(n, q, j)} \prod_{k=0}^{q-1}\left(\begin{array}{c}
j \\
l_{k}
\end{array}\right)\left(1-\eta_{k}\right)^{j-l_{k}} .
\end{aligned}
$$

As $l_{k} \leq j$ for all $0 \leq k \leq q-1$, we see that $n \leq q j$. The above sum is nonempty if and only if

$$
\frac{n}{q} \leq j \leq n
$$


Substituting into equation (2.10), we deduce that

$$
\begin{aligned}
\left.D_{u}^{n} e^{i u^{q}}\right|_{u=t} & =\left.e^{i t^{q}} D_{u}^{n} e^{i\left(u^{q}-t^{q}\right)}\right|_{u=t} \\
& =e^{i t^{q}} \sum_{\frac{n}{q} \leq j \leq n} \frac{i^{j}}{j !} n ! t^{q j-n} \sum^{(n, q, j)} \prod_{k=0}^{q-1}\left(\begin{array}{c}
j \\
l_{k}
\end{array}\right)\left(1-\eta_{k}\right)^{j-l_{k}} .
\end{aligned}
$$

Actually we eventually shall need a formula for $D_{t}^{n} e^{i \alpha t^{q}}$, where $\alpha \in \mathbb{R}$ is a constant. However, such a formula follows easily from the above formula for $D_{t}^{n} e^{i t^{q}}$. Select $\beta \in \mathbb{C}$ such that $\beta^{q}=\alpha$ and write $u=\beta t$. Then

$$
D_{t}^{n} e^{i(\beta t)^{q}}=\left.\beta^{n} D_{u}^{n} e^{i u^{q}}\right|_{u=\beta t} .
$$

By the above equation for $D_{u}^{n} e^{i u^{q}}$,

$$
\begin{aligned}
D_{t}^{n} e^{i \alpha t^{q}} & =\left.\beta^{n} D_{u}^{n} e^{i u^{q}}\right|_{u=\beta t} \\
& =e^{i \alpha t^{q}} \sum_{\frac{n}{q} \leq j \leq n} \frac{(i \alpha)^{j}}{j !} n ! t^{q j-n} \sum^{(n, q, j)} \prod_{k=0}^{q-1}\left(\begin{array}{c}
j \\
l_{k}
\end{array}\right)\left(1-\eta_{k}\right)^{j-l_{k}} .
\end{aligned}
$$

Next we obtain upper estimates for $\left|D_{t}^{n} e^{i \alpha t^{q}}\right|$ for each $t \in \mathbb{R}$ and $n \in \mathbb{N}$ when $\alpha \in \mathbb{R}$ and $q \in \mathbb{N}$ with $q \geq 2$.

Assume that $n / q \leq j \leq n$. If $|t| \geq 1$, then $|t|^{q j-n} \leq|t|^{q n-n}=|t|^{(q-1) n}$. If $|t| \leq 1$, then $|t|^{q j-n} \leq 1$. Thus for all $t \in \mathbb{R}$, we have

$$
|t|^{q j-n} \leq(\max (|t|, 1))^{(q-1) n}
$$

Since

$$
\left(\begin{array}{c}
j \\
l_{k}
\end{array}\right) \leq 2^{j} \quad \text { for } 1 \leq k \leq q-1 \text { and } \quad\left(\begin{array}{c}
j \\
l_{0}
\end{array}\right)=1
$$

we see that

$$
\prod_{k=0}^{q-1}\left(\begin{array}{c}
j \\
l_{k}
\end{array}\right) \leq 2^{(q-1) j}
$$

As $\left|1-\eta_{k}\right| \leq 2$ and $0 \leq l_{k} \leq j$,

$$
\left|1-\eta_{k}\right|^{j-l_{k}} \leq 2^{j-l_{k}} \quad \text { and } \quad\left|\prod_{k=0}^{q-1}\left(1-\eta_{k}\right)^{j-l_{k}}\right| \leq 2^{q j-n},
$$

where we have used that $\sum_{k=0}^{q-1} l_{k}=n$. It follows that

$$
\left|\prod_{k=0}^{q-1}\left(\begin{array}{c}
j \\
l_{k}
\end{array}\right)\left(1-\eta_{k}\right)^{j-l_{k}}\right| \leq 2^{(q-1) j} 2^{q j-n}=2^{(2 q-1) j-n} .
$$

It is an elementary combinatorial result that the number of ordered $(q-1)$-tuples of nonnegative integers $\left(l_{1}, \ldots, l_{q-1}\right)$ such that $\sum_{k=1}^{q-1} l_{k}=n-j$ is

$$
\left(\begin{array}{c}
n-j+q-2 \\
q-2
\end{array}\right)
$$


This estimate does not take into account that $l_{k} \leq j$ for $1 \leq k \leq q-1$. It follows that for $n / q \leq j \leq n$,

$$
\begin{aligned}
\left|\sum^{(n, q, j)} \prod_{k=0}^{q-1}\left(\begin{array}{c}
j \\
l_{k}
\end{array}\right)\left(1-\eta_{k}\right)^{j-l_{k}}\right| & \leq\left(\begin{array}{c}
n-j+q-2 \\
q-2
\end{array}\right) 2^{(2 q-1) j-n} \\
& \leq 2^{n-j+q-2} 2^{(2 q-1) j-n}=2^{2(q-1) j+q-2} .
\end{aligned}
$$

Our estimates imply that

$$
\begin{aligned}
\left|D_{t}^{n} e^{i \alpha t^{q}}\right| & \leq \sum_{\frac{n}{q} \leq j \leq n}|\alpha|^{j} \frac{n !}{j !(n-j) !}(n-j) !(\max (|t|, 1))^{(q-1) n} 2^{2(q-1) j+q-2} \\
& \leq 2^{q-2}(\max (|t|, 1))^{(q-1) n}\left(n-j_{*}\right) ! \sum_{\frac{n}{q} \leq j \leq n}\left(\begin{array}{c}
n \\
j
\end{array}\right)\left(|\alpha| 2^{2(q-1)}\right)^{j}
\end{aligned}
$$

where $j_{*}$ denotes the smallest positive integer $j$ such that $n / q \leq j \leq n$. By the binomial theorem,

$$
\sum_{\frac{n}{q} \leq j \leq n}\left(\begin{array}{c}
n \\
j
\end{array}\right)\left(|\alpha| 2^{2(q-1)}\right)^{j} \leq \sum_{0 \leq j \leq n}\left(\begin{array}{l}
n \\
j
\end{array}\right)\left(|\alpha| 2^{2(q-1)}\right)^{j}=\left(1+|\alpha| 2^{2(q-1)}\right)^{n}
$$

so

$$
\left|D_{t}^{n} e^{i \alpha t^{q}}\right| \leq 2^{q-2}(\max (|t|, 1))^{(q-1) n}\left(1+|\alpha| 4^{(q-1)}\right)^{n}\left(n-j_{*}\right) !
$$

We conclude that there exists a constant $C \geq 1$ such that for all $t \in \mathbb{R}$ and $n \in \mathbb{N}$,

$$
\left|D_{t}^{n} e^{i \alpha t^{q}}\right| \leq C^{n+1}(\max (|t|, 1))^{(q-1) n}\left(n-j_{*}\right) ! \leq C^{n+1}(\max (|t|, 1))^{(q-1) n} n !
$$

As a consequence we can verify the theorem.

Proof of Theorem 1.1. Let $p(t)=\sum_{m \in F} A_{m} e^{i m \omega t^{q}}$ for all $t \in \mathbb{R}$, where $F$ is a finite set of integers, $A_{m} \in \mathbb{C}$ for $m \in F, \omega>0, A_{0}=0$ and $q \geq 2$ is an integer. Our calculations above show that $p$ satisfies inequality (2.6) in Lemma 2.2. The boundedness of $x$ on intervals of the form $\left(-\infty, t_{0}\right]$ is clear because $\lim _{t \rightarrow-\infty} x(t)$ exists and is finite. If one applies Lemma 2.2 and uses Stirling's formula, the theorem follows.

Remark 2.4. In fact, with the aid of the advanced calculus form of Stirling's formula, one can replace $\left(n-j_{*}\right)$ ! in (2.11) with $(n !)^{1-1 / q}$.

It is obvious that $\left(n-j_{*}\right) !=1$ for $n=1,2$ because $q \geq 2$. Thus we can assume that $n \geq 3$.

Note that $n / q \leq j_{*}<n / q+1$, so if we choose $q_{*}>0$ such that $j_{*}=n / q_{*}$, then

$$
\frac{1}{q} \leq \frac{1}{q_{*}}<\frac{1}{q}+\frac{1}{n} \text { and } n-\frac{n}{q} \geq n-\frac{n}{q_{*}}>n-\frac{n}{q}-1 .
$$

Also, since $n \geq 3$ and $q \geq 2$, it is true that $n-n / q>1$.

By Stirling's formula,

$$
\left(n-j_{*}\right) !=\frac{\left(n\left(1-\frac{1}{q_{*}}\right)\right)^{n\left(1-\frac{1}{q_{*}}\right)}}{e^{n\left(1-\frac{1}{q_{*}}\right)}} \sqrt{2 \pi n\left(1-\frac{1}{q_{*}}\right)} \exp \left(\frac{\lambda\left(n\left(1-\frac{1}{q_{*}}\right)\right)}{12 n\left(1-\frac{1}{q_{*}}\right)}\right) .
$$


Since $n(1-1 / q)>1$ and $n(1-1 / q) \geq n\left(1-1 / q_{*}\right)$,

$$
\left(n\left(1-\frac{1}{q_{*}}\right)\right)^{n\left(1-\frac{1}{q_{*}}\right)} \sqrt{2 \pi n\left(1-\frac{1}{q_{*}}\right)} \leq\left(n\left(1-\frac{1}{q}\right)\right)^{n\left(1-\frac{1}{q}\right)} \sqrt{2 \pi n\left(1-\frac{1}{q}\right)} .
$$

Since

$$
e^{n\left(1-\frac{1}{q_{*}}\right)}>e^{n\left(1-\frac{1}{q}\right)-1},
$$

we conclude that

$$
\left(n-j_{*}\right) ! \leq e\left(\frac{n}{e}\right)^{n\left(1-\frac{1}{q}\right)}\left[\left(\frac{q-1}{q}\right)^{\left(\frac{q-1}{q}\right)}\right]^{n} \sqrt{2 \pi n\left(1-\frac{1}{q}\right)} \exp \left(\frac{\lambda\left(n\left(1-\frac{1}{q_{*}}\right)\right)}{12 n\left(1-\frac{1}{q_{*}}\right)}\right) .
$$

Stirling's formula for $n$ ! gives that

$$
\left(\frac{n}{e}\right)^{n\left(1-\frac{1}{q}\right)}=(n !)^{1-\frac{1}{q}}(2 \pi n)^{-\frac{1}{2}\left(1-\frac{1}{q}\right)} \exp \left(\frac{-\lambda(n)\left(1-\frac{1}{q}\right)}{12 n}\right) .
$$

Substituting for $(n / e)^{n\left(1-\frac{1}{q}\right)}$ gives that

$$
\left(n-j_{*}\right) ! \leq\left[\left(\frac{q-1}{q}\right)^{\left(\frac{q-1}{q}\right)}\right]^{n}(n !)^{1-\frac{1}{q}}(2 \pi n)^{\frac{1}{2 q}} \exp \left(1+\frac{\lambda\left(n\left(1-\frac{1}{q_{*}}\right)\right)}{12 n\left(1-\frac{1}{q_{*}}\right)}\right) .
$$

It follows, using our previous estimates for $\left|D_{t}^{n} e^{i \alpha t^{q}}\right|$, that there exists a constant $C_{*} \geq 1$ such that for all $t \in \mathbb{R}$ and $n \in \mathbb{N}$,

$$
\left|D_{t}^{n} e^{i \alpha t^{q}}\right| \leq C_{*}^{n+1}(\max (|t|, 1))^{(q-1) n}(n !)^{1-\frac{1}{q}} .
$$

\section{Acknowledgements}

R. Nussbaum was partially supported by NSF Grant DMS-1201328. G. Vas was supported by the Fulbright Program and by the Hungarian Scientific Research Fund, Grant No. K109782.

\section{References}

[1] M. Gevrey, Maurice, Sur la nature analytique des solutions des équations aux dérivées partielles. Premier mémoire (in French), Annales Scientifiques de l'École Normale Supérieure 35(1918), 129-190. url

[2] T. KRISzTIN, Analyticity of solutions of differential equations with a threshold delay, in: Recent Advances in Delay Differential Equations (Springer Proceedings in Mathematics \& Statistics 94, F. Hartung and M. Pituk eds., 2014), Springer, Cham, 2014, 173-180. MR3280191

[3] J. Mallet-Paret, R. D. Nussbaum, Analyticity and nonanalyticity of solutions of delaydifferential equations, SIAM J. Math. Anal. 46(2014), No. 4, 2468-2500. MR3229655

[4] J. Mallet-Paret, R. D. Nussbaum, Analytic solutions of delay-differential equations, in preparation. 
[5] J. Mallet-Paret, R. D. Nussbaum, Asymptotic homogenization of differential-delay equations and a question of analyticity, in preparation.

[6] R. D. Nussbaum, Periodic solutions of analytic functional differential equations are analytic, Michigan Math. J. 20(1973), 249-255. MR0322315

[7] T. Yamanaka, A new higher order chain rule and Gevrey class, Ann. Global Anal. Geom. 7(1989), No. 3, 179-203. MR1039118 